\title{
PREFERÊNCIA DE SAÚVA LIMÃO, Atta sexdens rubropilosa Forel, 1908 (HYMENOPTERA, FORMICIDAE) A DIFERENTES ESPÉCIES FLORESTAIS, EM CONDIÇÕES DE LABORATÓRIO
}

\author{
LEAF CUTTING ANT, Atta sexdens rubropilosa Forel, 1908 (HYMENOPTERA, FORMICIDAE) \\ PREFERENCE FOR DIFFERENT SPECIES UNDER LABORATORY CONDITIONS
}

\author{
Otávio Peres Filho ${ }^{1}$ Alberto Dorval ${ }^{2}$ Evônio Berti Filho ${ }^{3}$ \\ RESUMO
}

Avaliou-se a preferência de saúva limão, Atta sexdens rubropilosa Forel, 1908, para 41 espécies florestais nativas e exóticas, em condições de laboratório. As espécies mais transportadas foram gmelina (Gmelina arborea), leucena (Leucaena leucocephala), pau-ferro (Caesalpinia ferrea), albizia (Albizia lebbeck) e orelha-de-negro (Enterolobium contortisiliquum) e as menos transportadas foram eucalipto (Eucalyptus grandis), jequitibá (Cariniana strellensis) e mutamba (Guazuma tomentosa).

Palavras-chave: formiga cortadeira, preferência por espécies florestais, Atta sexdens rubropilosa.

\begin{abstract}
It was evaluated the cutting ant preference of Atta sexdens rubropilosa Forel, 1908, to native and exotic forest tree species, in laboratory conditions. The most preferred species were: gmelina (Gmelina arborea), leucaena (Leucaena leucocephala), caesalpinia (Caesalpinia ferrea), albizzia (Albizia lebbeck) and enterolobium (Enterolobium contortisiliquum) and the less preferred ones were: eucalypt (Eucalyptus grandis), jequitiba (Cariniana strellensis ) and guazuma (Guazuma tomentosa).
\end{abstract}

Key words: leaf-cutting ant, preference for forest species, Atta sexdens rubropilosa.

\section{INTRODUÇÃO}

Dentre as formigas cortadeiras, destaca-se o gênero Atta como um dos mais nocivos a um grande número de culturas agrícolas e florestais no território brasileiro, pois dos insetos desfolhadores, é considerado o grupo mais importante, por causa dos grandes danos que causam e a quantidade de recursos envolvidos no seu controle (Mariconi, 1970). Na seleção do hospedeiro, as saúvas, aparentemente, são influenciadas por repelentes químicos presentes nas plantas e por mudanças físicas nas folhas, associadas às flutuações de temperatura (Waller, 1986). Estudos voltados para a ação de substâncias repelentes têm sido realizados no sentido de isolar os componentes responsáveis, visando a aplicação prática no manejo das saúvas cortadeiras. Os lipídeos apresentam propriedades inibitórias às espécies Atta cephalotes e Acromyrmex octospinous sendo que os extratos cerosos obtidos de folhas jovens são menos repelentes do que os obtidos de folhas velhas (Littledyke e Cherret, 1978). Substâncias voláteis como trans-beta-ocimeno [(E)-3,7-Demeter-1,3,6-octatrieno] obtido de Astronium graveolens (Anacardiaceae) são repelentes para Atta laevigata (Chent et al., 1984). Jaisson et al. (1984) verificaram, em testes de laboratório e de campo com diferentes espécies vegetais, que o epóxido cariofileno, um dos repelentes para A. cephalotes, é um potente fungicida mesmo quando empregado em pequenas quantidades. Substâncias como o piplaroxide e dimetoxipiplantine obtidas de Piper tuberculatum demonstraram significativa ação de repelência para Atta cephalotes em bioensaio de laboratório (Capron \& Wiemer, 1996). Barnola et al. (1997) mostraram que os terpenos voláteis de Pinus caribaea são responsáveis pelas diferentes intensidades de forrageamento de

1. Engenheiro Florestal, Dr., Professor Adjunto do Departamento de Engenharia Florestal, Faculdade de Engenharia Florestal, Universidade Federal do Mato Grosso, CEP 78060-900, Cuiabá (MT). o.peres@zaz.com.br

2. Engenheiro Florestal, Doutorando em Entomologia pela Universidade Federal do Paraná, Técnico de Nível Superior do Departamento de Engenharia Florestal, Faculdade de Engenharia Florestal, Universidade Federal do Mato Grosso, CEP 78060-900, Cuiabá (MT). adorval@ zaz.com.br

3. Engenheiro Agrônomo, Dr., Professor Titular do Departamento de Entomologia, Patologia e Zoologia Agrícola, Escola Superior de Agricultura Luiz de Queiroz, Universidade de São Paulo, CEP 13418-900, Piracicaba (SP). eberti@carpa.ciagri.usp.br

Recebido para publicação em 22/05/2001 e aceito em 8/08/2002. 
A.laevigata. Substâncias como o flavonóide quercetina podem ser responsáveis pela seleção de Atta sexdens rubropilosa, sobretudo, em concentrações mais elevadas, como constatado por Sugayama e Salatino (1997). Salatino et al. (1998) demonstraram que triflavonóide lupeol, um dos compostos formadores das ceras da epicutícula de Didymopanax vinosum (Araliaceae), é um importante deterrente para Atta sexdens rubropilosa.

Considerando-se a potencialidade econômica de uso de diversas espécies florestais nativas e exóticas para reflorestamentos, homogêneos ou não bem como daquelas espécies já cultivadas, avaliou-se, no presente trabalho, a preferência de saúva limão, Atta sexdens rubropilosa, por diferentes espécies florestais, em condições de laboratório, a fim de fornecer subsídios ao manejo dessa espécie.

\section{MATERIAL E MÉTODOS}

O experimento foi realizado no Laboratório de Proteção Florestal, da Faculdade de Engenharia Florestal, da Universidade Federal de Mato Grosso, sob temperatura média $27 \pm 2^{\circ} \mathrm{C}$ e umidade relativa de $70 \pm 10 \%$. Para os testes de atratividade foram empregadas 41 espécies florestais (Tabela 1).

TABELA 1: Relação das espécies florestais empregadas nos testes de prefer ência de saúva limão, Atta sexdens rubropilosa Forel, 1908.

TABLE 1: Forest tree species used for testing the preference of the leaf cutting ant Atta sexdens rubropilosa Forel, 1908.

\begin{tabular}{ll|ll}
\hline Nome Vulgar & \multicolumn{1}{c|}{ Espécie } & Nome Vulgar & \multicolumn{1}{c}{ Espécie } \\
\hline açoita-cavalo & Luehea divaricata & jequitibá & Cariniana strellensis \\
albizia & Albizia lebbeck & leucena & Leucaena leucocephala \\
almecega & Protium heptaphyllum & louro branco & Cordia glabrata \\
angico vermelho & Anadenanthera peregrina & mirindiba & Terminalia glabrescens \\
aricá & Physocalina scaberrimum & mogno & Swietenia macrophylla \\
aroeira & Astronium urundeuva & murici & Birsonima verbascifolia \\
cambará & Vochysia divergens & mutamba & Guazuma tomentosa \\
cedro & Cedrela fissilis & tamboriu & Enterolobium contortisiliquum \\
cerejeira & Amburana acreana & paratudo & Tabebuia carahyba \\
copaíba & Copaiba langsdorffii & paricá & Schyzolobium amazonicum \\
cumbaru & Dipteryx alata & pau-de-bicho & Terminalia argentea \\
eritrina & Erythrina mulungu & pau-ferro & Caesalpinia ferrea \\
eucalipto & Eucalyptus grandis & pau-terra & Qualea grandiflora \\
genipapo & Genipa americana & pequi & Caryocar brasiliensis \\
gmelina & Gmelina arborea & seringueira & Hevea brasiliensis \\
gonçaleiro & Astronium fraxinifolium & sete-cascas & Phitecolobium samam \\
guatambu & Aspidosperma sp. & tarumã & Vitex cymosa \\
ingá & Inga edulis & teca & Tectona grandis \\
ipê rosa & Tabebuia avellanedae & timbó & Magonia pubescens \\
jacarandá & Jacaranda cuspidifolia & unha-de-vaca & Bauhinia variegata \\
jatobá & Hymenaea courbaril & & \\
\hline
\end{tabular}

Foi utilizada a metodologia de Cherret e Seaforth (1970), modificada, utilizando-se placas de alumínio de $15 \mathrm{~cm}$ x $15 \mathrm{~cm}$ com 100 quadrículas de $1,0 \mathrm{~cm} \times 1,0 \mathrm{~cm}$ e bordaduras de $2,5 \mathrm{~cm}$. As placas foram devidamente lavadas com sabão neutro e esterilizadas em estufa a $150^{\circ} \mathrm{C}$, pelo período de 1 hora, antes e após qualquer utilização. Em cada placa, foram colocadas 25 amostras provenientes de folhas jovens, obtidas da parte apical dos ramos do terço superior da copa, e maduras, obtidas da parte basal dos ramos do terço inferior da copa. As amostras foram feitas com um vazador metálico de forma circular, com diâmetro de $1 \mathrm{~cm}$. Cada espécie florestal foi considerada um tratamento e cada placa uma repetição, empregando-se cinco repetições para cada tratamento. No primeiro teste, foram oferecidas, simultaneamente, às saúvas 21 espécies florestais, sendo que cada repetição foi disposta ao acaso em uma arena cercada em suas laterais; em um segundo teste, foram empregadas mais vinte espécies florestais, totalizando 41 tratamentos. Os 
tratamentos foram disponibilizados às saúvas por um período de 10 minutos ou até que a penúltima amostra de qualquer um dos tratamentos tivesse sido carregada. Foram realizados mais dois testes sendo um com as espécies florestais menos transportadas e outro com as espécies florestais mais transportadas, dos primeiro e segundo testes. A formiga utilizada nos testes foi a saúva limão, Atta sexdens rubropilosa Forel, 1908, provenientes de um formigueiro mantido em laboratório e as folhas empregadas nos testes obtidas de árvores adultas desenvolvidas a pleno sol. $\mathrm{O}$ formigueiro permaneceu 48 horas sem receber qualquer tipo de folha antes de iniciar os testes. A atratividade foi avalia da determinando-se a quantidade de discos foliares transportados de cada tratamento, sendo os dados analisados pelo teste de Scott Knott ao n ível de 5\%.

\section{RESULTADOS E DISCUSSÃO}

Nas figuras 1, 2, 3 e 4, as espécies florestais seguidas da mesma letra não diferiram estatisticamente entre si. No primeiro teste, gmelina foi a espécie mais transportada, vindo a teca em segundo lugar e aroeira, cerejeira e tarumã em terceiro, as demais espécies não diferiram estatisticamente entre si (Figura 1). O jatobá, Hymenaea courbaril, citado por Anjos et al. (1998) como planta imune ao ataque de formigas cortadeiras, apresentou pequena quantidade de discos foliares transportados (Figura 1), confirmando

A

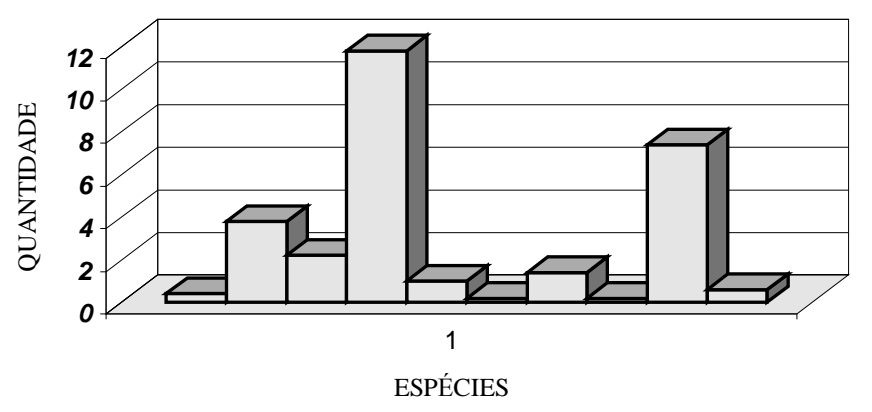

B

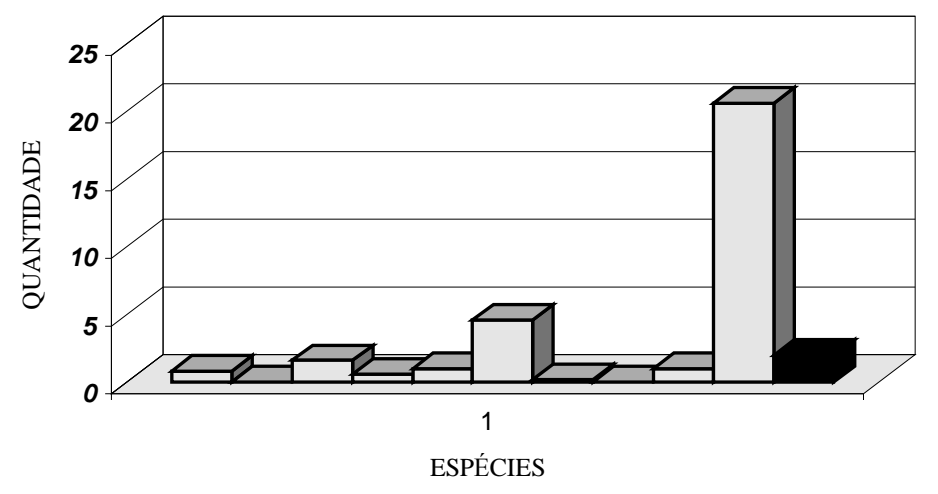

$\square$ jatobá (d)

$\square$ cerejeira (c)

$\square$ ipê (d)

$\square$ teca (b)

$\square$ cedro (d)

$\square$ cambará (d)

$\square$ jequitibá (d)

$\square$ copaíba (d)

$\square$ aroeira (c)

Dlouro branco (d)

$\square$ seringueira (d)

$\square$ almecega (d)

$\square$ ingá (d)

$\square$ pequi (d)

$\square$ mogno (d)

$\square$ tarumã (c)

$\square$ cumbarú (d)

$\square$ eucalipto (d)

$\square$ pau-de-bicho (d)

$\square$ gmelina (a)

açoita-cavalo (d)

FIGURA 1: Quantidade média de discos foliares de diferentes espécies florestais transportados por saúva-limão, Atta sexdens rubropilosa Forel, 1908, em condições de laboratório. Primeiro teste (A e B). Espécies seguidas da mesma letra, entre parênteses, não diferem entre si ao nível de 5\% pelo teste de Scott Knott.

FIGURE 1: Average number of leaf discs from different forest tree species carried by the leaf cutting ant, Atta sexdens rubropilosa Forel, 1908, in laboratory conditions. First test (A and B). Forest tree species followed by the same letter, in parenthesis, do not differ at the 5\% level by the Scott Knott test. 
os resultados obtidos por esses autores. Para Hubbel e Wiemer (1983) o repelente volátil epóxido cariofileno, encontrado em Hymenaea courbaril, evita o corte das folhas por Atta. No segundo teste as espécies mais transportadas foram albizia, leucena e sete-cascas, seguidas de guatambu, tamboriu, pau-ferro e as demais não diferindo estatisticamente entre si (Figura 2). Para o teste efetuado com as espécies mais transportadas dos primeiro e segundo testes, gmelina, leucena e pau-ferro foram as espécies mais transportadas sendo seguida por albizia, guatambu e tamboriu (Figura 2). Apesar da teca ter apresentado destaque no primeiro teste, sendo superada apenas por gmelina, no teste das mais transportadas aparece em último lugar (Figura 3).

A

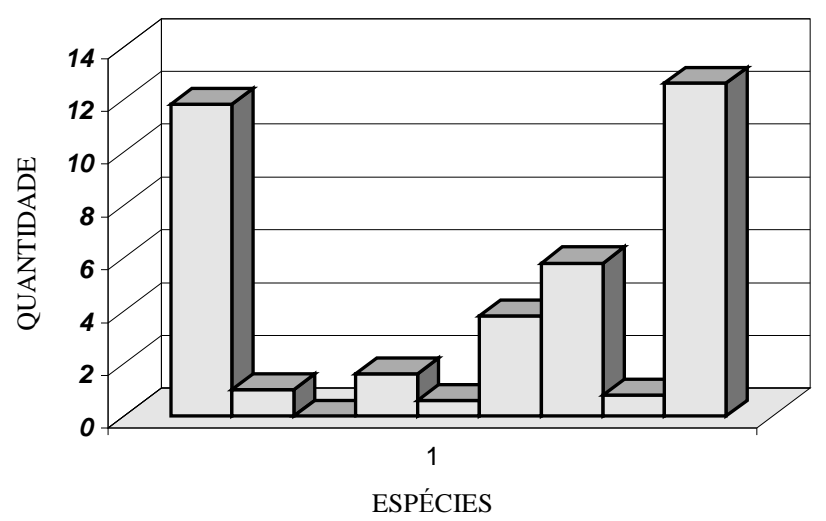

B

ESPÉCIES

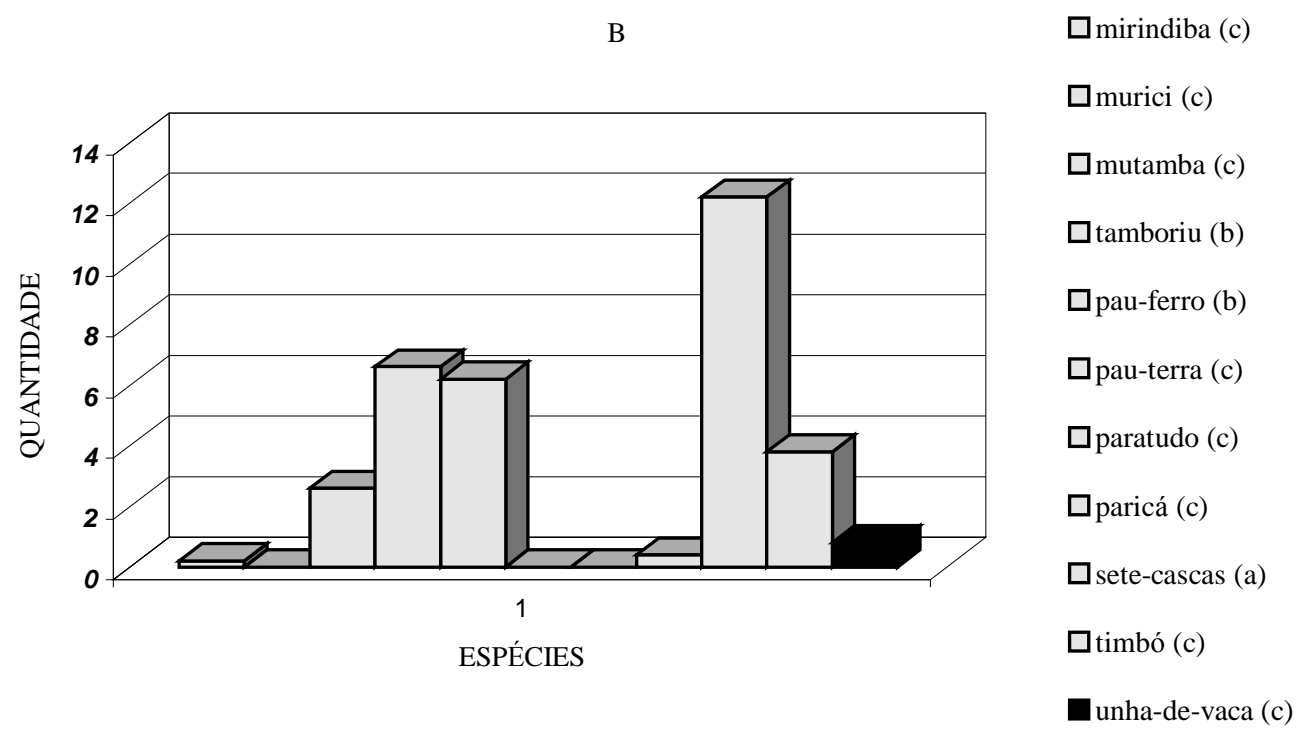

$\square$ albizia (a)

$\square$ angico (c)

$\square$ aricá (c)

Deritrina (c)

$\square$ genipapo (c)

$\square$ gonçaleiro(c)

$\square$ guatambú (b)

$\square$ jacarandá (c)

Dleucena (a)

FIGURA 2: Quantidade média de discos foliares de diferentes espécies florestais transportados por saúva-limão, Atta sexdens rubropilosa Forel, 1908, em condições de laboratório. Segundo teste (A e B). Espécies seguidas da mesma letra, entre parênteses, não diferem entre si ao nível de 5\% pelo teste de Scott Knott

FIGURE 2: Average number of leaf discs from different forest tree species carried by the leaf cutting ant, Atta sexdens rubropilosa Forel, 1908, in laboratory conditions Second test (A and B). Forest tree species followed by the same letter, between bracnets, do not differ at the $5 \%$ level by the Scott Knott test. 
Para as espécies menos transportadas dos dois primeiros testes e submetidas a um novo teste, obteve -se que as espécies menos transportadas foram eucalipto, sendo seguida de jequitibá, mutamba e seringueira (Figura 4).

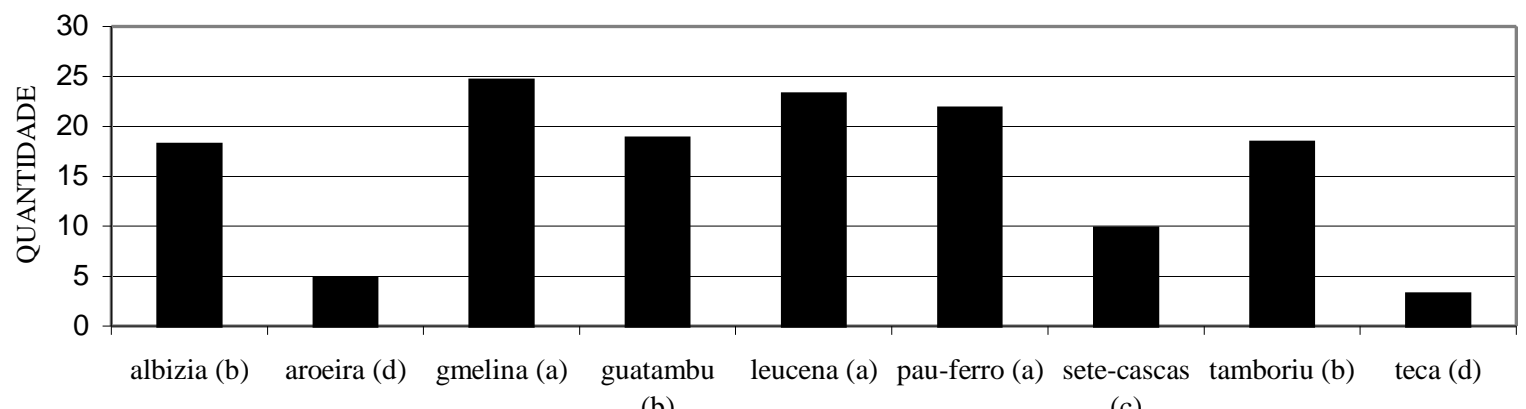

(b)

(c)

ESPÉCIES

FIGURA 3: Quantidade média de discos foliares das espécies florestais mais transportadas nos primeiros testes com saúva-limão, Atta sexdens rubropilosa Forel, 1908, em condições de laboratório. Espécies seguidas da mesma letra, entre parênteses, não diferem entre si ao nível de $5 \%$ pelo teste de Scott Knott.

FIGURE 3: Average number of the preferred forest tree species leaf discs carried by the leaf cutting ant Atta sexdens rubropilosa Forel, 1908 in laboratory conditions: Forest tree species followed by the same letter, between bracnets, do not differ at the5\% level by the Scott Knott test.

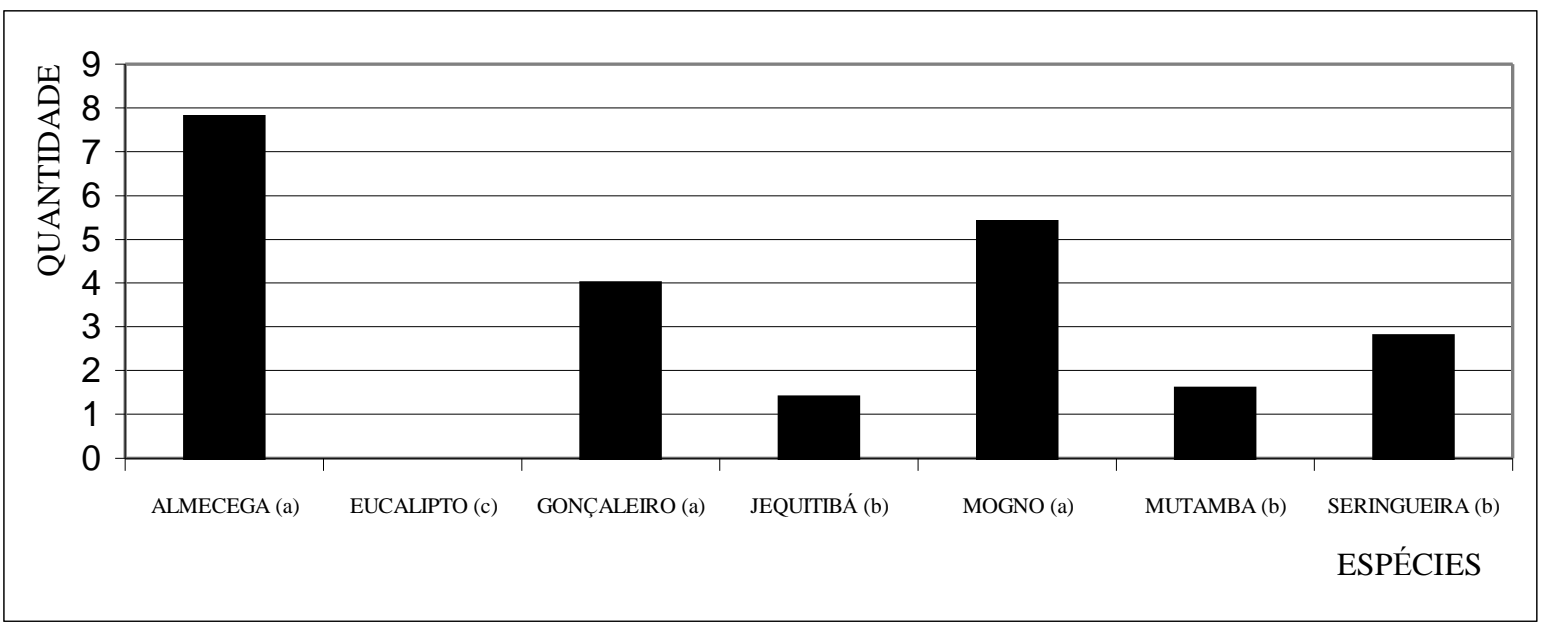

FIGURA 4: Quantidade média de discos foliares das espécies florestais menos transportadas nos primeiros testes com saúva-limão, Atta sexdens rubropilosa Forel, 1908, em condições de laboratório. Espécies seguidas da mesma letra, entre parênteses, não diferem entre si ao nível de 5\% pelo teste de Scott Knott.

FIGURE 4: Average number of the less preferred forest tree species leaf discs carried by the leaf cutting ant Atta sexdens rubropilosa Forel, 1908 in laboratory conditions: Forest tree species followed by the same letter, between bracnets, do not differ at the5\% level by the Scott Knott test .

Os resultados obtidos possibilitam avaliar que, dependendo da oferta de espécie apresentada, o comportamento da saúva pode ser alterado, como constatado em teca submetida inicialmente e em uma segunda vez, apresentando, uma situação de última colocada, todavia para gmelina o fato foi repetido 
evidenciando a maior preferência de Atta sexdens rubropilosa por essa espécie florestal (Figura 3). Observações de campo também demonstraram que gmelina é severamente atacada por Atta sexdens rubropilosa, no município de Cuiabá/MT, mesmo quando presente em arboretos formado por diferentes espécies florestais. Observações de campo também demonstraram severos ataques de Atta sexdens rubropilosa em mudas de teca no campo, chegando essas formigas a se deslocarem da mata nativa para cortar folhas do reflorestamento com teca. Schoereder (1985) também constatou que as plantas menos preferidas no laboratório foram as menos procuradas no campo. Os testes de carregamento de folhas em condições de laboratório fornecem informações parciais importantes sobre a atratividade, todavia outras situações tais como idade das árvores testadas, sazonalidade, posições das folhas na arquitetura da copa das árvores ou, ainda, como estudado por Folgarait et al. (1996) em situações de sombreamento a pleno sol. Estudos posteriores deverão ser realizados visando à obtenção de compostos repelentes e atraentes, com o propósito de subsidiar o manejo integrado de praga, bem como o cons órcio de diferentes espécies florestais. the same letter, in parenthesis, do not differ at the $5 \%$ level by the Scott Knott test.

\section{CONCLUSÕES}

De acordo com os resultados obtidos pode-se concluir que as espécies Gmelina arborea e Caesalpinia ferrea foram as mais atrativas ao ataque de Atta sexdens rubropilosa, quando comparada com as demais espécies, do grupo das espécies mais transportadas. Para o grupo das espécies menos transportadas a espécie Eucalyptus grandis foi a menos atrativa sendo seguida pelas espécies Guazuma tomentosa e Cariniana strellensis.

\section{AGRADECIMENTOS}

Ao Prof. Dr. Carlos Alberto Moraes Passos e ao Engenheiro Agrônomo Fernando Tadeu Caseiro, da Faculdade de Engenharia Florestal/UFMT e da Faculdade de Agronomia e Medicina Veterinária/UFMT respectivamente, pelas análises estatísticas realizadas. Ao Técnico de Laboratório Manoel Lauro da Silva pelo inestimável auxílio no desenvolvimento deste trabalho.

\section{REFERÊNCIAS BIBLIOGRÁFICAS}

ANJOS, N.; DELLA LUCIA, T.M.C.; MAYHÉ-NUNES, A.J. Guia prático sobre formigas cortadeiras em reflorestamentos. Ponte Nova: Editora Graff Cor, 1998. 97p.

BARNOLA, L.F.; CEDENO, A.; HASEGAWA, M. Intraindividual variations of volatile terpene contents in Pinus caribaea needles and its possible relationship to Atta laevigata herbivory. Biochemical Sysytematics and Ecology, v. 25, n. 8, p. 707-716, 1997.

CAPRON, M.A.; WIEMER, D.F. Piplaroxide, an ant-repellente piperidine epoxide from Piper tuberculatum. Journal of Natural Products, v. 59, n. 8, p. 794-795, 1996.

CHENT, T.K.; WIEMER, D.F.; HOWARD, J.J. A volatile leafcutter ant repellent from Astronium graveolens. Naturwissenschaften, v. 71, n. 2, p. 97-98, 1984.

CHERRET, J.M.; SEAFORTH, C.E. Phytochemical arrestants for leaf-cutting ants, Atta cephalotes (L.) and Acromyrmex octospinosus (Reich), with some notes on the ants responses. Bull.Ent.Res., v. 59, p. 615-625, 1970.

FOLGARAIT, P.J.; DYER, L.A.; MARQUIS, R.J.; BRAKER, H.E. Leaf cutting ant preferences for five native tropical plantation tree species growing under different light conditions. Entomologia Experimentalis et Applicata, v. 80, n. 3, p. 521-530, 1996.

HUBBEL, S.P.; WIEMER, D.F. Host plant selection by an attine ant. In: JAISSON, P. (Ed.) Social insects in the tropics. Paris: Université Paris-Nord, 1983. p. 133-153.

JAISSON, P.; HUBBELL, S.P.; WIEMER, D.F. Host plant selection by an attine ant. In: FIRST INTERNATIONAL SYMPOSIUM ORGANIZED BY THE INTERNATIONAL UNION FOR THE STUDY OF SOCIAL INSECTS AND THE SOCIEDAD MEXICANA DE ENTOMOLOGIA, 1980, México. Proceedings... Mexico, 1984 v. 2, p. 133-154.

LITTLEDYKE, M.; CHERRET, J.M. Defense mechanisms in young and old leaves against cutting by the leaf-cutting ants Atta cephalotes (L.) and Acromyrmex octospinosus (Reich) (Hymenoptera, Formicidae). Bulletin of Entomological Research, v. 68, n. 2, p. 263-271, 1978. 
MARICONI, F.A.M. As saúvas. São Paulo: Editora Agronômica Ceres, 1970. 167p.

SALATINO, A.; SUGAYAMA, R.L.; NEGRI, G.; VILELAS, W. Effect of constituents of the foliar wax of Didymopanax vinosum on the foragin activity of the leaf-cutting ant Atta sexdens rubropilosa. Entomologia Experimentalis et Applicata, v. 86, n. 3, p. 261-266, 1998.

SCHOEREDER, J.H. Aspectos ecológicos da saúva no cerrado. 1985. 112p. Dissertação (Mestrado em Ciências) Universidade de São Paulo, São Paulo.

SUGAYAMA, R.L.; SALATINO, A. Influence of rutin and quercetin on substrate selection by the leaf-cutting ant Atta sexdens rubropilosa. Revista Brasileira de Biologia, v. 57, n. 1, p. 121-125, 1997.

WALLER, D.A. The foraging ecology of Atta texana in Texas. In: LOFGREN, C.S.; VANDERMEER R.K. (eds.). Fire ants and leaf-cutting ants: biology and management. Boulder: Westveew Press, p. 146-158. 